\title{
Neoadjuvant Chemotherapy Followed by Radical Surgery versus Radiotherapy (with or without Chemotherapy) in Patients with Stage IB2, IIA, or IIB Cervical Cancer: A Systematic Review and Meta-Analysis
}

\author{
Qingjian Ye $\mathbb{D}$, Yuebo Yang $\mathbb{D}$, Xinran Tang, Jing Li, Xiaomao Li $\mathbb{D}$, and Yu Zhang $\mathbb{D}$ \\ Department of Gynecology, The Third Affiliated Hospital of Sun Yat-sen University, Guangzhou 510630, China \\ Correspondence should be addressed to Xiaomao Li; lixmao@mail.sysu.edu.cn and Yu Zhang; zhangyu6@mail.sysu.edu.cn
}

Received 9 August 2019; Accepted 12 June 2020; Published 27 July 2020

Academic Editor: Zhongjie Shi

Copyright (C) 2020 Qingiian Ye et al. This is an open access article distributed under the Creative Commons Attribution License, which permits unrestricted use, distribution, and reproduction in any medium, provided the original work is properly cited.

Background. This study was to compare the efficacy and safety between neoadjuvant chemotherapy followed by radical surgery $(\mathrm{NACT}+\mathrm{RS})$ and radiotherapy only (RT) or concurrent chemoradiotherapy (CCRT) for treatment of patients with stage IB2, IIA, or IIB cervical cancer. Method. The electronic databases of PubMed, Embase, and the Cochrane Library were searched to screen relevant studies from their inception to October 2018. Clinical data including overall survival (OS), disease-free survival (DFS), and adverse events were extracted. Egger's test was used to evaluate the publication bias, and sensitivity analysis was conducted to estimate the robustness of results. Results. Finally, three randomized controlled trials (RCTs) and two case-control studies consisting of 1,275 patients with stage IB2, IIA, or IIB cervical cancer were included in the current study. Overall, pooled results showed no significant differences in OS ( (hazard ratio $(\mathrm{HR})=0.603,95 \% \mathrm{CI}=0.350-1.038)$ and $\mathrm{DFS}(\mathrm{HR}=0.678,95 \%$ $\mathrm{CI}=0.242-1.904)$ for patients treated with NACT+RS compared with RT only or CCRT, but the subgroup analysis showed that the OS and DFS were significantly longer in the NACT+RS groups than the RT or CCRT group (OS: $\mathrm{HR}=0.431,95 \% \mathrm{CI}=$ $0.238-0.781, p=0.006$; DFS: $\mathrm{HR}=0.300,95 \% \mathrm{CI}=0.187-0.482, p<0.001$ ) for the population with median follow-up time of more than 60 months. For adverse events, the incidence of thrombocytopenia in the NACT+RS group was significantly higher than that in the RT only or CCRT group (relative risk $(\mathrm{RR})=3.240,95 \%$ CI 1.575-6.662), while the incidence of diarrhea was significantly lower than that in the RT only or CCRT group ( $R R=0.452,95 \% \mathrm{CI}=0.230-0.890)$. Conclusion. These findings suggest that the short-term therapeutic effects of the two treatments may be possibly equal for patients with stage IB2-IIB cervical cancer, but the long-term effects for improving OS and DFS may be better using NACT+RS compared with the RT only or CCRT.

\section{Introduction}

Cervical cancer is the second most common malignant tumor in women and has become a major public health problem worldwide $[1,2]$. In developing countries, more than $80 \%$ of patients with cervical cancer are diagnosed at an advanced stage, which seriously affects the prognosis of the sufferers [3]. Locally advanced cervical cancer (LACC) is defined as IB2-IIB stages according to the International Federation of Gynecology and Obstetrics (FIGO) staging system [4], treatment of which may reduce the risk of progression and subsequent death $[5,6]$. Since 2000, concurrent chemo- radiotherapy (CCRT) is recommended as the standard treatment for LACC in some countries [7,8], which has been demonstrated to prolong the survival by approximately $50 \%$ compared with radiotherapy (RT) alone [5, 6]. However, a meta-analysis revealed that there were still $25 \%$ to $40 \%$ of patients with LACC experiencing relapse and the time to metastases at 5 years was also not significantly improved after CCRT compared with RT [9], implying the necessity to explore more effective therapeutic strategies.

Neoadjuvant chemotherapy (NACT) followed by radical surgery (RS) has been extensively explored over the past decade as it could reduce the requirement of postoperative 
radiotherapy and thus improve the prognosis $[10,11]$. NACT can shrink tumor size and hence transform inoperable into radically resected tumors [12]. Also, via a systematic review and meta-analysis of six randomized controlled trials (RCTs) (1,072 women), Rydzewska et al. found that the combination of NACT and RS was superior to surgery alone in improving progression-free survival (hazard ratio $(\mathrm{HR})=0.76,95 \%$ confidence intervals $(\mathrm{CI})=0.62$ to $0.94, p=0.01]$ for LACC patients [13]. A meta-analysis of five RCTs performed by Peng et al. also suggested that NACT+RS could reduce the risk of lymph node metastasis, interstitial infiltration, vascular infiltration, and positive incision margin compared to the RS-alone group, which were all prognostic-related factors [14]. These findings indicated that NACT+RS may be an attractive alternative treatment option for LACC, in addition to CCRT or RT.

However, whether the therapeutic effect of NACT+RS is better than RT alone or CCRT remains unclear because controversial conclusions were reported by previous studies. For example, Gupta et al. observed that the 5-year disease-free survival (DFS) in the NACT+RS group was lower than that of the CCRT group $(69.3 \%$ vs. $76.7 \%, p=0.038)$, whereas the corresponding 5-year overall survival (OS) was significant different between two groups [15]. Yin et al. proved that the NACT+RS group had significantly higher survival rates than the CCRT group (DFS: $\mathrm{HR}=3.535, p<0.0001$; OS: $\mathrm{HR}=3.157, p<0.0001$ ) [16]. These differences may be attributed to a small sample size of an individual study. Therefore, it is indispensable to comprehensively evaluate the effects of these therapeutic strategies for patients with LACC in the current study using a systematic review and meta-analysis.

\section{Methods}

2.1. Search Strategy. The electronic databases of PubMed, Embase, and Cochrane were systematically searched to screen the relevant studies up to October 2018. In addition, the references included in the searched literatures and related reviews were also searched to prevent eligible articles from being omitted. The search terms included (1) "cervical cancer" or "uterine cancer"; and (2) "neoadjuvant chemotherapy" or "preoperative chemotherapy"; and (3) "radiotherapy" or "chemoradiotherapy". The language was not limited when literature searching was performed.

2.2. Inclusion and Exclusion Criteria. Studies that met the following criteria were included in this study. (1) Subjects were cervical cancer patients with stage IB2-IIB. (2) Patients in the study group underwent NACT+RS. (3) Patients in the control group underwent RT only or CCRT. (4) OS, DFS, or both were used as the study endpoint. (5) DFS- or OS-related data can be extracted. Detailed exclusion criteria were listed as follows. (1) Study subjects were noncervical cancer. (2) Studies were descriptive such as case reports, reviews, and guidelines. (3) Study endpoint was not about survival time. (4) Studies did not compare the effects of NACT+RS with RT or CCRT. (5) Study subjects were not patients with stage IB2-IIB cervical cancer. Two researchers independently searched and screened the literatures. If there was an inconsistency between the two researchers, a third investigator was enrolled to resolve it.

2.3. Data Extraction and Quality Assessment. Data extraction was also independently carried out by two researchers based on preestablished forms. Considering that patients with cervical cancer of stage III or more serious stages are not suitable for surgery, this study only compared the efficacy of patients with IB2-IIB cervical cancer. OS and DFS were taken as the primary objective, and the adverse events were used as secondary research results. Basic characteristics (including author, publication year, country, design, FIGO stage, NACT method, and follow-up time from studies that met the inclusion criteria) were extracted. If the OS or DFS data in the study could not be extracted directly, Engauge Digitizer 4.1 was used to extract the corresponding data through Kaplan-Meier survival curves. Adverse events in this study mainly included anemia, thrombocytopenia, neutropenia, leukopenia, vomiting, diarrhea, and neurotoxicity. The quality of the included studies in this meta-analysis was evaluated according to the guidelines in the Cochrane Handbook for Systematic Reviews of Interventions in Chapter 8 [17]. This study was approved by the ethics committee of the Third Affiliated Hospital of Sun Yat-sen University.

2.4. Statistical Analysis. Stata statistical software (version 14.0, Stata Corporation, College Station, TX, USA) was used for statistical analysis and Review Manager (version 5.3.3, Cochrane Collaboration, Oxford, UK) was used for quality evaluation of literatures. Summary HR and corresponding 95\% CI were calculated from pooled data for OS and DFS, while the risk ratio (RR) value and the corresponding $95 \%$ $\mathrm{CI}$ were computed to compare adverse events between the $\mathrm{NACT}+\mathrm{RS}$ group and the RT or CCRT group. Cochran's $Q$ test and $I^{2}$ statistic were involved to quantify the heterogeneity of the included studies. If the $I^{2}$ value was greater than $50 \%$, the heterogeneity was considered to be obvious and thus the random-effects model was used for the metaanalysis; otherwise, a fixed effects model was applied. Egger's test was used to assess the publication bias. A sensitivity analysis was performed by using the "remove one study" method to evaluate the impact of individual studies on overall results.

\section{Results}

3.1. Study Selection and Quality Assessment. Detailed literature search and screening processes are shown in Figure 1. A total of 1,266 related articles were retrieved, including 1,256 directly obtained through searching PubMed, Embase, and Cochrane Library databases and 10 articles after reading the references or related reviews. Of these, 903 studies were removed due to duplicate literatures. Among the left 363 articles, 358 articles were further excluded because they were case reports $(n=29), 134$ articles of noncervical cancers $(n=134)$, descriptive studies $(n=26)$, unrelated topics $(n=114)$, systematic reviews and meta-analyses $(n=8)$, neoadjuvant chemotherapy and no surgery $(n=7)$, nonprognosis $(n=18)$, early cervical cancer $(n=7)$, and without 


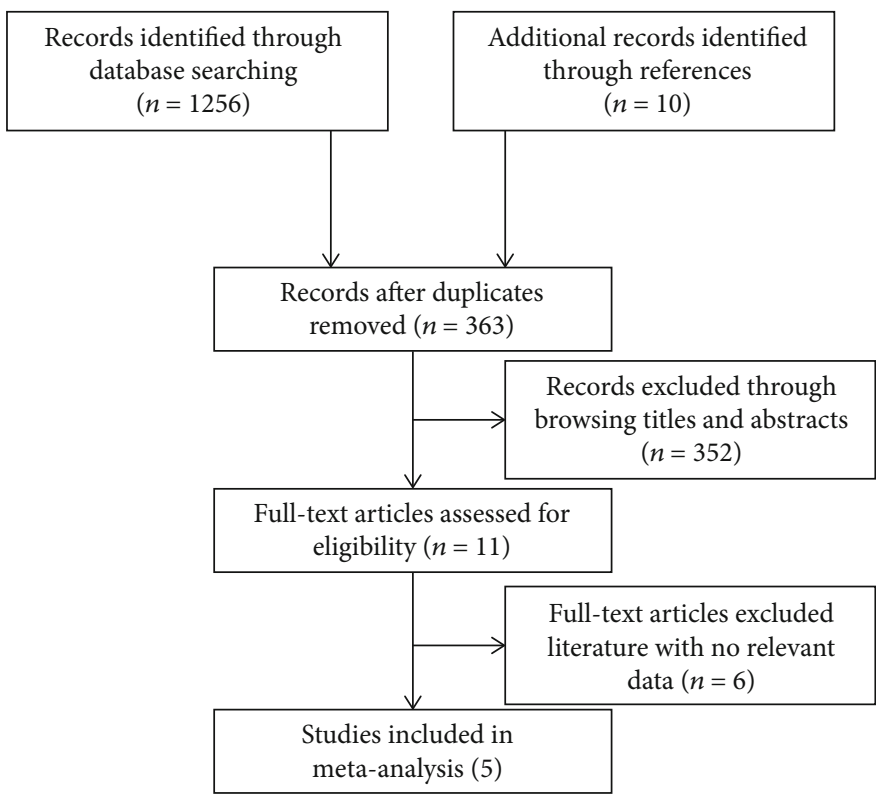

FIgURE 1: Flow diagram of the entire research literature.

neoadjuvant $(n=9)$. Finally, five articles that met the inclusion criteria were enrolled in the current study.

3.2. Study Characteristics and Quality Assessment. These five studies included two case-control studies and three RCTs and were published from 1998 to 2018 [15, 16, 18-20]. The main characteristics of the studies are listed in Table 1. A total of 1,275 patients with stage IB2-IIB cervical cancer were involved in this study. In order to identify the quality of the included studies, the Cochrane Collaboration risk of bias tool was used. Two studies did not describe the information of random sequence generation and the allocation concealment, and three studies did not use a blinded mode to assess the outcome. The detailed quality evaluation results are shown in Figure 2.

3.3. Pooled Analysis for OS. All of the five studies compared the OS outcome of NACT+RS with RT or CCRT for cervical cancer patients with stage IB2-IIB [15, 16, 18-20]. A random effects model was chosen for meta-analysis because of the large degree of heterogeneity between studies $\left(I^{2}=74.5 \%\right.$, $p=0.004)$. The pooled results showed that compared to RT or CCRT, NACT+RS did not significantly extend the OS of patients with stage IB2-IIB cervical cancer $(\mathrm{HR}=0.603,95 \% \mathrm{CI}=0.350-1.038 ; \mathrm{p}=0.068)$ (Figure 3$)$.

3.4. Pooled Analysis for DFS. Four of the included studies reported DFS in 1,034 patients with stage IB2-IIB cervical cancer, with a median follow-up of 39.0-82.8 months [15, $16,19]$. Similar to OS, there was a large heterogeneity in DFS; therefore, a random effects model $\left(I^{2}=93.5 \%, p<\right.$ $0.001)$ was performed for summary analysis. The summary results also showed no difference in DFS between the $\mathrm{NACT}+\mathrm{RS}$ group and the RT or CCRT group, indicating that NACT + RS could not increase DFS $(\mathrm{HR}=0.678,95 \% \mathrm{CI}=$ $0.242-1.904 ; p<0.001$ ) (Supplemental figure 1).
3.5. Subgroup Analysis. In order to explore the source of heterogeneity among studies, a subgroup meta-analysis was conducted according to country, publication year, sample size, follow-up time, study design, and control therapy. As illustrated in Table 2, the OS and DFS of patients who undertook NACT+RS were found to be significantly longer than that of the RT or CCRT group (OS: HR $=0.431,95 \%$ $\mathrm{CI}=0.238-0.781, p=0.006$; DFS: $\mathrm{HR}=0.300,95 \% \mathrm{CI}=$ $0.187-0.482, p<0.001)$ in the population with median follow-up time of more than 60 months, indicating the $\mathrm{NACT}+\mathrm{RS}$ may be more effective than the RT or CCRT group for improving the long-term survival for cervical cancer patients with stage IB2-IIB. The same situation was identified in studies designed as case-control trials (Table 2: OS: $\mathrm{HR}=0.302,95 \% \mathrm{CI}=0.170-0.538, p<0.001$; Table 3: DFS: $\mathrm{HR}=0.300,95 \% \mathrm{CI}=0.187-0.482, p<0.001)$.

3.6. Adverse Events. In order to compare the safety of the two treatment methods, a pooled analysis of the adverse events was undertaken. No significant differences in anemia, neutropenia, leukopenia, vomiting, or neurotoxicity was observed between the two groups (Supplemental table 1). However, the incidence of thrombocytopenia was significantly higher in the NACT+RS group than in the RT or CCRT group $(\mathrm{RR}=3.240,95 \% \mathrm{CI}=1.575-6.662, p=$ 0.001 ), while the RT or CCRT group was more prone to develop diarrhea than the NACT+RS group $(\mathrm{RR}=0.452$, $95 \% \mathrm{CI}=0.230-0.890, p=0.022)$.

3.7. Publication Bias and Sensitivity Analysis. Potential publication bias of the included studies was investigated using the Egger test. As a result, no obvious publication bias was found for OS and DFS (OS: $p=0.131$; DFS: $p=0.461$ ). Additionally, a sensitivity analysis was performed to investigate the impact of individual study on the results of the pooled analysis. The result showed that a single study had little effect on 
TABLE 1: The main characteristics of the five included studies.

\begin{tabular}{|c|c|c|c|c|c|c|c|c|c|c|c|c|}
\hline \multirow{2}{*}{ Study } & \multirow{2}{*}{ Year } & \multirow{2}{*}{ Country } & \multirow{2}{*}{ Design } & \multicolumn{2}{|c|}{$\begin{array}{l}\text { Case } \\
\text { group }\end{array}$} & \multicolumn{3}{|c|}{ Control group } & \multirow{2}{*}{$\begin{array}{l}\text { FIGO } \\
\text { stage }\end{array}$} & \multirow{2}{*}{ NACT } & \multirow{2}{*}{$\begin{array}{l}\text { Follow-up } \\
\text { (month) }\end{array}$} & \multirow{2}{*}{$\begin{array}{l}\text { Primary } \\
\text { endpoints }\end{array}$} \\
\hline & & & & $n$ & Age & $n$ & Age & Therapy & & & & \\
\hline Gupta [16] & 2018 & India & RCT & 316 & 50 & 317 & 48 & CCRT & IB2-IIB & $\begin{array}{l}3 \text { cycles of paclitaxel } \\
\text { and carboplatin }\end{array}$ & 58.5 & OS,DFS \\
\hline Abou-Taleb [17] & 2016 & Japan & $\begin{array}{c}\text { Case } \\
\text { control }\end{array}$ & 70 & 53 & 22 & 77 & RT & IB2-IIB & $\begin{array}{l}\text { 1-3 cycles of irinotecan } \\
\text { and nedaplatin }\end{array}$ & Unclear & OS \\
\hline Yin [18] & 2011 & China & $\begin{array}{c}\text { Case } \\
\text { control }\end{array}$ & 187 & 43 & 94 & 47 & CCRT & IB2-IIB & 2 or 3 cycles of cisplatin & 82.8 & OS,DFS \\
\hline Chang [19] & 2000 & Taiwan & RCT & 68 & 46 & 52 & 47 & $\mathrm{RT}$ & IB2-IIA & 3 cycles of VBP scheme ${ }^{\#}$ & 39.0 & OS,DFS \\
\hline Sardi [20] & 1998 & Argentina & RCT & 76 & $44^{*}$ & 73 & $41.5^{*}$ & RT & IIB & 3 cycles of VBP scheme & 84.0 & OS \\
\hline
\end{tabular}

Abbreviations: FIGO: International Federation of Gynecology and Obstetrics; NACT: neoadjuvant chemotherapy; RS: radical surgery; CCRT: concurrent chemoradiotherapy; RT: radiotherapy; OS: overall survival; DFS: disease-free survival; *mean age. Age values are presented as median; follow-up time is presented as median. ${ }^{8}$ NACT was performed using transuterine arterial chemotherapy (TUAC) or intravenous. ${ }^{*}$ The VBP scheme was used ( 3 courses of vincristine $1 \mathrm{mg} / \mathrm{m}^{2}$ on day 1 , bleomycin $25 \mathrm{mg} / \mathrm{m}^{2}$ on days 1 to 3 , and cisplatinum $50 \mathrm{mg} / \mathrm{m}^{2}$ on day 1 , at 10 -day intervals).

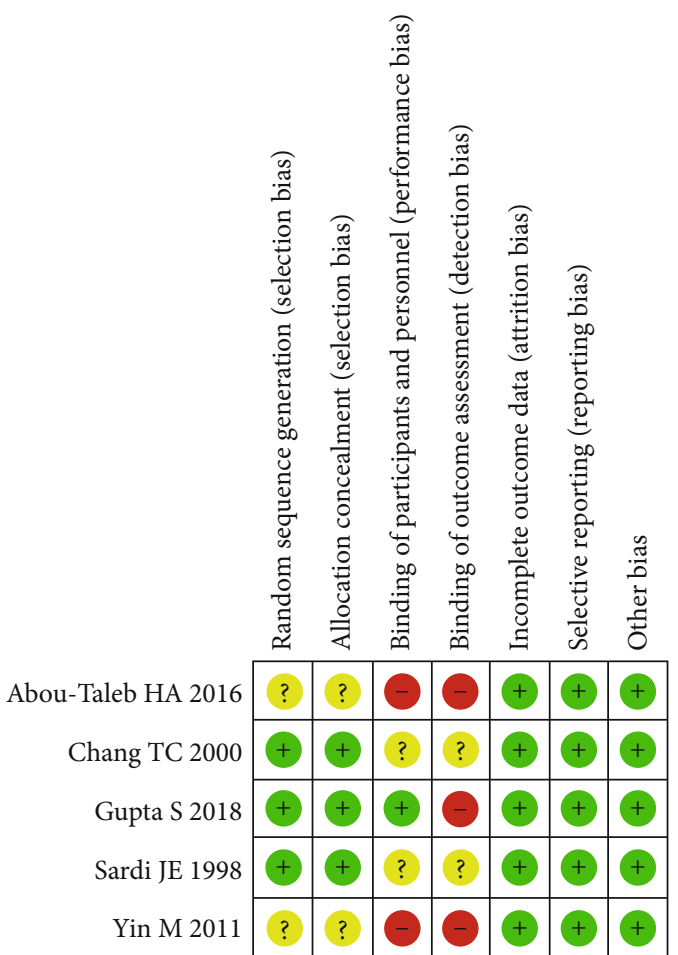

Figure 2: Risk of bias summary for the five included studies.

the summary results, which indicated that the results of this study were robust (Supplemental figure 2).

\section{Discussion}

This meta-analysis compared the efficacy of NACT+RS with RT or CCRT for patients with stage IB2-IIB cervical cancer by summarizing the results of five studies. The results suggested no differences in OS and DFS between the two groups. These results seemed to be inconsistent with the study of Tierney who also used five studies to conduct a pooled analysis to compare the effects of
NACT+RS with RT only [11] and the results showed that $\mathrm{NACT}+\mathrm{RS}$ could prolong the OS and DFS compared with RT only in patients with cervical cancer. These differences may be attributed to the fact that stage III patients were excluded in our study as stage III patients are usually not allowed to undergo surgery. It is worth noting that for LACC, the US standard treatment is CCRT $[5,6,21$, 22], while some institutions in Europe and Asia are $\mathrm{NACT}+\mathrm{RS}$ [23-25]. In subgroup analysis, the impact of $\mathrm{NACT}+\mathrm{RS}$ versus CCRT on survival was also compared. Similarly, no differences in OS and DFS were identified between patients using these two treatments. However, we found that the OS and DFS of the NACT+RS group were longer compared with the CCRT group for patients with a median follow-up of more than 60 months, indicating the long-term effects of NACT+RS may be more superior. But, it should be noted that the studies included in this meta-analysis was not large enough, and more multicenter studies with longer follow-up are needed to verify this conclusion.

Adverse events, which are highly linked to a specific situation (whether the course of treatment can be completed on schedule), are one of the issues that must be considered during treatment. According to the report of Abou-Taleb et al., patients treated with irinotecan/nedaplatin for NACT are less likely to suffer from severe anemia and vomiting than those receiving CCRT [18]. Our study did not find that the neoadjuvant therapy could reduce the incidence of severe anemia and vomiting compared with the RT or CCRT group in patients with stage IB2IIB cervical cancer. Pignata and coworkers argued that diarrhea was the only severe and dose-limiting nonhematologic toxicity associated with CCRT [26]. This study demonstrated that the incidence of diarrhea in the CCRT or RT group was higher than that in the NACT+RS group, which was consistent with that of Pignata et al. [26].

Our meta-analysis had many advantages. This was the first study to evaluate the efficacy and safety of NACT+RS versus RT only or concurrent CCRT in patients with stage IB2, IIA, or IIB cervical cancer. Because cervical cancer 


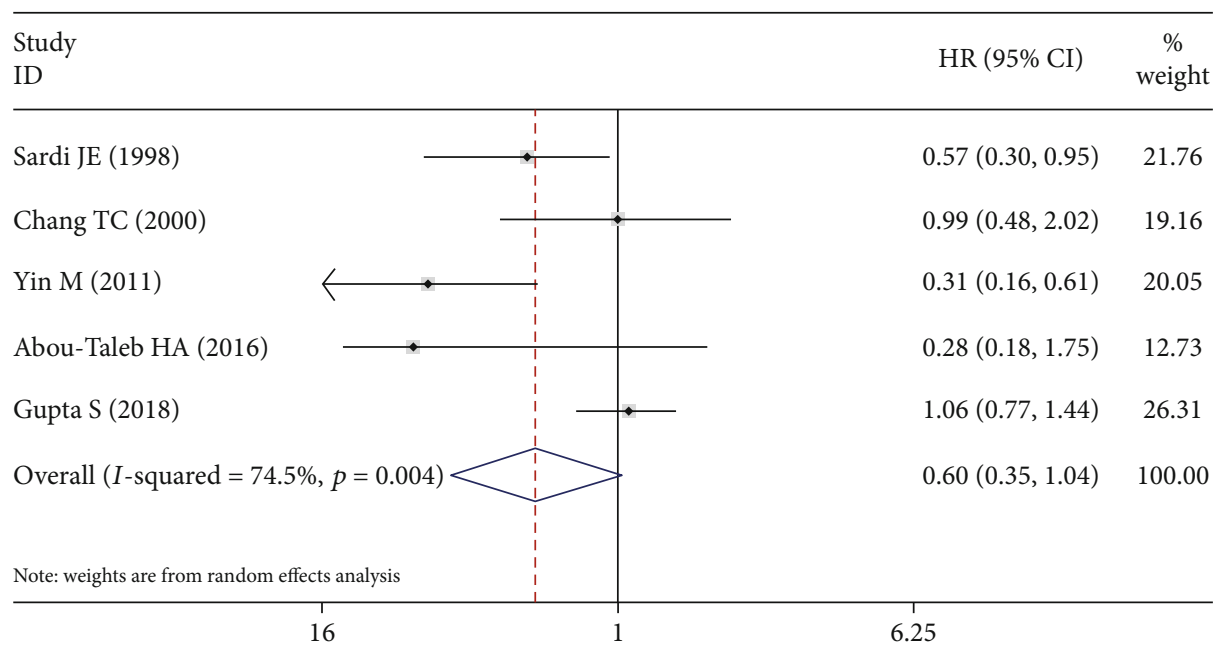

FIGURE 3: Forest plots for the overall survival.

TABLE 2: The results of pooled HR for OS in different subgroups.

\begin{tabular}{|c|c|c|c|c|c|c|}
\hline & No. & HR & $95 \% \mathrm{CI}$ & $P$ & $I$-squared & $p$ \\
\hline \multicolumn{7}{|l|}{ Country } \\
\hline Asian & 4 & 0.598 & $0.295-1.212$ & 0.154 & $79.2 \%$ & 0.004 \\
\hline Other & 1 & 0.570 & $0.320-1.014$ & 0.056 & - & - \\
\hline \multicolumn{7}{|l|}{ Publication year } \\
\hline Before 2010 & 2 & 0.719 & $0.422-1.227$ & 0.227 & $27.5 \%$ & 0.240 \\
\hline After 2010 & 3 & 0.488 & $0.179-1.325$ & 0.159 & $85.8 \%$ & 0.001 \\
\hline \multicolumn{7}{|l|}{ Sample size } \\
\hline$<150$ & 3 & 0.602 & $0.332-1.092$ & 0.095 & $19.7 \%$ & 0.166 \\
\hline$>150$ & 2 & 0.595 & $0.179-1.980$ & 0.397 & $90.6 \%$ & 0.001 \\
\hline \multicolumn{7}{|l|}{ Follow-up time } \\
\hline$\leq 60$ & 2 & 1.049 & $0.787-1.397$ & 0.746 & $0.0 \%$ & 0.864 \\
\hline$>60$ & 2 & 0.431 & $0.238-0.781$ & 0.006 & $45.3 \%$ & 0.176 \\
\hline Unclear & 1 & 0.280 & $0.090-0.873$ & 0.028 & - & - \\
\hline \multicolumn{7}{|l|}{ Study design } \\
\hline RCT & 3 & 0.877 & $0.595-1.291$ & 0.505 & $42.4 \%$ & 0.176 \\
\hline Case control & 2 & 0.302 & $0.170-0.538$ & 0.000 & $0.0 \%$ & 0.880 \\
\hline \multicolumn{7}{|l|}{ Controls therapy } \\
\hline CCRT & 2 & 0.595 & $0.179-1.980$ & 0.397 & $90.6 \%$ & 0.001 \\
\hline RT & 3 & 0.603 & $0.332-1.092$ & 0.095 & $44.2 \%$ & 0.166 \\
\hline
\end{tabular}

Abbreviations: CCRT: concurrent chemoradiotherapy; RT: radiotherapy.

patients with stage IB2-IIB are often available for surgery, this evaluation is particularly clinically instructive. In addition, we conducted quality assessments, publication biases, and sensitivity analyses on the included studies, which ensured the reliability of the results. At the same time, this study had some limitations. First, only five articles were included in this meta-analysis; therefore, the number of documents was relatively small. Second, this study only compared the survival rate and adverse events but did not compare the quality of life due to the lack of relevant report data. The quality of life is also a key factor in the choice of treatment. In 2013, Le Borgne and colleagues studied the long-term health-related quality of life in cervical cancer and found that quality of life of survivors receiving radiation therapy was significantly worse in terms of sexual dysfunction, urination and abdominal symptoms, and lymphedema compared with survivors who only received surgery [27].

In conclusion, this systematic review and meta-analysis suggests that the short-term therapeutic effects of the two treatments may be possibly equal for patients with stage IB2-IIB cervical cancer, but the long-term effects on OS and DFS may be better using NACT+RS compared with the RT only or CCRT. NACT+RS may be especially applicable in 
TABLE 3: The results of pooled HR for DFS in different subgroups.

\begin{tabular}{|c|c|c|c|c|c|c|}
\hline & No. & HR & $95 \% \mathrm{CI}$ & $P$ & $I$-squared & $p$ \\
\hline \multicolumn{7}{|l|}{ Country } \\
\hline Asian & 3 & 0.678 & $0.242-1.904$ & 0.461 & $93.5 \%$ & 0.000 \\
\hline Other & 0 & - & - & - & - & - \\
\hline \multicolumn{7}{|l|}{ Publication year } \\
\hline Before 2010 & 1 & 0.690 & $0.370-1.288$ & 0.244 & - & - \\
\hline After 2010 & 2 & 0.669 & $0.142-3.154$ & 0.611 & $96.7 \%$ & 0.000 \\
\hline \multicolumn{7}{|l|}{ Sample size } \\
\hline$<150$ & 1 & 0.690 & $0.370-1.288$ & 0.244 & - & - \\
\hline$>150$ & 2 & 0.669 & $0.142-3.154$ & 0.611 & $96.7 \%$ & 0.000 \\
\hline \multicolumn{7}{|l|}{ Follow-up time } \\
\hline$\leq 60$ & 2 & 1.057 & $0.511-2.187$ & 0.882 & $77.6 \%$ & 0.035 \\
\hline$>60$ & 1 & 0.300 & $0.187-0.482$ & 0.000 & - & - \\
\hline \multicolumn{7}{|l|}{ Study design } \\
\hline RCT & 2 & 1.057 & $0.511-2.187$ & 0.882 & $77.6 \%$ & 0.035 \\
\hline Case control & 1 & 0.300 & $0.187-0.482$ & 0.000 & - & - \\
\hline \multicolumn{7}{|l|}{ Control therapy } \\
\hline CCRT & 2 & 0.669 & $0.142-3.154$ & 0.611 & $96.7 \%$ & 0.000 \\
\hline $\mathrm{RT}$ & 1 & 0.690 & $0.370-1.288$ & 0.244 & - & - \\
\hline
\end{tabular}

Abbreviations: CCRT: concurrent chemoradiotherapy; RT: radiotherapy.

some areas where radiotherapy equipment is in short supply. This meta-analysis provides the basis for the patients with cervical cancer to choose the better treatment plan and calls for the increase of radiotherapy equipment.

\section{Data Availability}

The data supporting this meta-analysis are from previously reported studies and datasets, which have been cited.

\section{Conflicts of Interest}

No potential conflicts of interest were disclosed.

\section{Authors' Contributions}

Qingjian Ye and Yuebo Yang contributed in the topic selection, literature screening, data extraction, and paper writing. Xinran Tang and Jing Li contributed in the statistical analysis and literature quality evaluation. Yu Zhang and Xiaomao Li guided the topic selection and article writing. Qingjian Ye and Yuebo Yang contributed equally to this work.

\section{Supplementary Materials}

Supplementary 1 Supplemental Figure 1 Forest plots for the DFS. Supplementary 2 Supplemental Figure 2 Publication bias and sensitivity analysis for OS and DFS. (A) Publication bias for overall survival. (B) Publication bias for disease-free survival. (C) Sensitivity analysis for overall survival. (D) Sensitivity analysis for disease-free survival. (Supplementary Materials)

\section{References}

[1] WHO, "World Health Organization Report: comprehensive cervical cancer control: a guide to essential practice. World Health Organization web site, 2006," February 2008, http:// www.who.int/reproductive-health/publications/cervical_ cancer_gep/index.htm.

[2] J. Ferlay, I. Soerjomataram, R. Dikshit et al., "Cancer incidence and mortality worldwide: sources, methods and major patterns in GLOBOCAN 2012," International Journal of Cancer, vol. 136, no. 5, pp. E359-E386, 2015.

[3] A. P. Vizcaino, V. Moreno, F. X. Bosch et al., "International trends in incidence of cervical cancer: II. Squamous-cell carcinoma," International Journal of Cancer, vol. 86, no. 3, pp. 429435, 2000.

[4] J. Prat and FIGO Committee on Gynecologic Oncology, "Staging classification for cancer of the ovary, fallopian tube, and peritoneum," International Journal of Gynaecology and Obstetrics, vol. 124, no. 1, pp. 1-5, 2014.

[5] B. J. Monk, K. S. Tewari, and W. J. Koh, "Multimodality therapy for locally advanced cervical carcinoma: state of the art and future directions," Journal of Clinical Oncology, vol. 25, no. 20, pp. 2952-2965, 2007.

[6] G. M. Thomas, "Improved treatment for cervical cancerconcurrent chemotherapy and radiotherapy," The New England Journal of Medicine, vol. 340, no. 15, pp. 1198-1200, 1999.

[7] J. A. Green, J. M. Kirwan, J. F. Tierney et al., "Survival and recurrence after concomitant chemotherapy and radiotherapy for cancer of the uterine cervix: a systematic review and metaanalysis," The Lancet, vol. 358, no. 9284, pp. 781-786, 2001.

[8] H. Lukka, H. Hirte, A. Fyles et al., "Concurrent Cisplatin-based Chemotherapy plus Radiotherapy for Cervical Cancer - a 
Meta-analysis," Clinical Oncology, vol. 14, no. 3, pp. 203-212, 2002.

[9] Chemoradiotherapy for Cervical Cancer Meta-Analysis Collaboration, "Reducing uncertainties about the effects of chemoradiotherapy for cervical cancer: a systematic review and meta-analysis of individual patient data from 18 randomized trials," Journal of Clinical Oncology, vol. 26, no. 35, pp. 58025812, 2008.

[10] F. Landoni, E. Sartori, T. Maggino et al., "Is there a role for postoperative treatment in patients with stage Ib2-IIb cervical cancer treated with neo-adjuvant chemotherapy and radical surgery? An Italian multicenter retrospective study," Gynecologic Oncology, vol. 132, no. 3, pp. 611-617, 2014.

[11] J. Tierney and Neoadjuvant Chemotherapy for Locally Advanced Cervical Cancer Meta-Analysis Collaboration, "Neoadjuvant chemotherapy for locally advanced cervical cancer: a systematic review and meta-analysis of individual patient data from 21 randomised trials," European Journal of Cancer, vol. 39, no. 17, pp. 2470-2486, 2003.

[12] P. Naga Ch, L. Gurram, S. Chopra, and U. Mahantshetty, "The management of locally advanced cervical cancer," Current Opinion in Oncology, vol. 30, no. 5, pp. 323-329, 2018.

[13] L. Rydzewska, J. Tierney, S. Burdett, and P. R. Symonds, "Neoadjuvant chemotherapy plus surgery versus surgery for cervical cancer," Cochrane Database of Systematic Reviews, vol. 12, no. 12, 2012.

[14] Y. H. Peng, X. X. Wang, J. S. Zhu, and L. Gao, "Neo-adjuvant chemotherapy plus surgery versus surgery alone for cervical cancer: meta-analysis of randomized controlled trials," Journal of Obstetrics and Gynaecology Research, vol. 42, no. 2, pp. 128135, 2016.

[15] S. Gupta, A. Maheshwari, P. Parab et al., "Neoadjuvant chemotherapy followed by radical surgery versus concomitant chemotherapy and radiotherapy in patients with stage IB2, IIA, or IIB squamous cervical cancer: a randomized controlled trial," Journal of Clinical Oncology, vol. 36, no. 16, pp. 15481555, 2018.

[16] M. Yin, F. Zhao, G. Lou et al., "The long-term efficacy of neoadjuvant chemotherapy followed by radical hysterectomy compared with radical surgery alone or concurrent chemoradiotherapy on locally advanced-stage cervical cancer," International Journal of Gynecological Cancer, vol. 21, no. 1, pp. 9299, 2011.

[17] J. P. T. Higgins and S. Green, Cochrane Handbook for Systematic Reviews of Interventions Version 5.1.0 [updated March 2011], The Cochrane Collaboration, 2011, http://www .cochrane-handbook.org.

[18] H. A. Abou-Taleb, M. Koshiyama, N. Matsumura et al., "Clinical efficacy of neoadjuvant chemotherapy with irinotecan (CPT-11) and nedaplatin followed by radical hysterectomy for locally advanced cervical cancer," The Journal of International Medical Research, vol. 44, no. 2, pp. 346-356, 2016.

[19] T. C. Chang, C. H. Lai, J. H. Hong et al., "Randomized trial of neoadjuvant cisplatin, vincristine, bleomycin, and radical hysterectomy versus radiation therapy for bulky stage IB and IIA cervical cancer," Journal of Clinical Oncology, vol. 18, no. 8, pp. 1740-1747, 2000.

[20] J. E. Sardi, C. E. Sananes, A. A. Giaroli et al., "Neoadjuvant chemotherapy in cervical carcinoma stage IIB: a randomized controlled trial," International Journal of Gynecological Cancer, vol. 8, no. 6, pp. 441-450, 1998.
[21] M. Morris, P. J. Eifel, J. Lu et al., "Pelvic radiation with concurrent chemotherapy compared with pelvic and para-aortic radiation for high-risk cervical cancer," The New England Journal of Medicine, vol. 340, no. 15, pp. 1137-1143, 1999.

[22] P. G. Rose, B. N. Bundy, E. B. Watkins et al., "Concurrent cisplatin-based radiotherapy and chemotherapy for locally advanced cervical cancer," The New England Journal of Medicine, vol. 340, no. 15, pp. 1144-1153, 1999.

[23] H. Chen, C. Liang, L. Zhang, S. Huang, and X. Wu, "Clinical efficacy of modified preoperative neoadjuvant chemotherapy in the treatment of locally advanced (stage IB2 to IIB) cervical cancer: randomized study," Gynecologic Oncology, vol. 110, no. 3, pp. 308-315, 2008.

[24] N. Katsumata, H. Yoshikawa, H. Kobayashi et al., "Phase III randomised controlled trial of neoadjuvant chemotherapy plus radical surgery_vs_radical surgery alone for stages IB2, IIA2, and IIB cervical cancer: a Japan Clinical Oncology Group trial (JCOG 0102)," British Journal of Cancer, vol. 108, no. 10, pp. 1957-1963, 2013.

[25] A. Gadducci, E. Sartori, T. Maggino et al., "Pathological response on surgical samples is an independent prognostic variable for patients with stage Ib2-IIb cervical cancer treated with neoadjuvant chemotherapy and radical hysterectomy: an Italian multicenter retrospective study (CTF study)," Gynecologic Oncology, vol. 131, no. 3, pp. 640-644, 2013.

[26] S. Pignata, P. Frezza, S. Tramontana et al., "Phase I study with weekly cisplatin-paclitaxel and concurrent radiotherapy in patients with carcinoma of the cervix uteri," Annals of Oncology, vol. 11, no. 4, pp. 455-459, 2000.

[27] G. Le Borgne, M. Mercier, A.-S. Woronoff et al., "Quality of life in long-term cervical cancer survivors: a population-based study," Gynecologic Oncology, vol. 129, no. 1, pp. 222-228, 2013. 